\title{
Measuring perceived process efficiency by employing customer satisfaction methodology
}

\section{Dag Bergsjö*}

Chalmers University of Technology,

SE-41296 Gothenburg, Sweden

Fax: +46317721375

Email: dagb@chalmers.se

*Corresponding author

\section{Lars-Uno Roos}

Karlstad University,

SE-65188 Karlstad, Sweden

Email: lars_uno_roos@yahoo.se

\begin{abstract}
This paper presents a novel methodology by which lean principles can be measured and analysed in a product development context. The methodology is based on customer satisfaction index calculations and analyses quantitative data to measure the performance of the engineering processes from an engineer's perspective. The quantitative data have been collected using an online questionnaire and been classified and analysed using partial least square (PLS) analysis. This paper presents the methodology, including the 18 lean principles identified, together with a case study performed at a large R\&D company. The questionnaire was completed by 242 employees. The study shows that the quantitative analysis points out relevant areas for improvement that are also qualitatively verified. The PLS analysis provides a rich material that can be explored to identify strong correlations between lean principles and desired business performance indicators. The case study singled out factors named 'product assurance practices' and 'process simplicity' as major improvement areas with a high impact on business performance.
\end{abstract}

Keywords: effectiveness; performance KPI; cost-effectiveness; information management; PLS analysis; lean principles; systems engineering process; cross-functional collaboration.

Reference to this paper should be made as follows: Bergsjö, D. and Roos, L-U. (2016) 'Measuring perceived process efficiency by employing customer satisfaction methodology', Int. J. Lean Enterprise Research, Vol. 2, No. 2, pp.95-114.

Biographical notes: Dag Bergsjö is an Associate Professor in the Systems Engineering and Engineering Design research group at Chalmers University of Technology. He received his $\mathrm{PhD}$ in Product and Production Development from in 2009. He is currently conducting research within the area of Lean product development, systems engineering and platform development (technology, product and production platforms). He is also interested in the integration of lean product development and product data/lifecycle management (PDM/PLM). 
Lars-Uno Roos was one of the first in Sweden to do research in the area of Lean Production. In 1990, he received his $\mathrm{PhD}$ with the thesis 'Japanization in production systems. Some case studies of total quality management in British manufacturing industry'. He has been head of quality in production, purchasing and product development in Swedish global industry companies.

\section{Introduction}

Process efficiency is a hot topic in industry today. It is often said that companies should spend less time and money to build better products. This belief places demands on management to implement and improve the processes continuously. Within the systems engineering community, there is a set of accepted indicators for assessing program and technical effectiveness (Rhodes et al., 2009). However, marketing teaches us that customer beliefs and expectations can also be measured and assessed (Ringle et al., 2010). The principle of measuring design efficiency and connecting it to management principles has been further supported by such researchers as Chiva and Alegre (2009).

Accurately measuring the efficiency of the product development process in an objective and effective manner is probably not feasible. From an organisational viewpoint, product realisation takes place in highly complex development settings typically involving large companies with a network of suppliers where engineers with different backgrounds are required to develop new and innovative products. From a technical perspective the products, consisting of components and software, are based on a variety of technologies and need to be flexible and scalable to fit into several ranges of product lines.

A challenge inherent in measuring process efficiency is the identification of the variables that are important to measure and the kind of engineering behaviour that is encouraged by such measurement. Some indicators are easy to measure, but their impact on actual business performance would be difficult to trace. Other measured aspects are highly significant, such as revenue per product or overall return on R\&D investments, but these are difficult to connect to specific parts of the development process, and it is in particular difficult to specify why a specific outcome has been achieved. The processes are dependent on a number of organisational and technical variables that need to be accounted for when evaluating the impact of different variables on efficiency. Also, the subjective viewpoint of a few managers or experts may not be correct and often lacks justification.

The main purpose of this paper is to use a statistical methodology from marketing with which to measure customer satisfaction in order to connect satisfaction with the development process to performance of the product realisation process. This methodology would complement the technical and financial indicators by collecting the engineers' opinions on the product realisation process focused on the perceived efficiency of the process and tracing it to principles based on important quality targets. The methodology is presented in general terms and then tested as a single case study at a company in the aerospace industry. 


\section{Research methodology}

This paper is based on an explorative research methodology using quantitative and qualitative methods to identify correlations between different performance indicators and principles of development.

The overall hypothesis is that "customer satisfaction measurements in marketing can be converted to a development setting to measure engineering process satisfaction". With this main hypothesis as a foundation, we aim to identify and verify correlations between qualitatively identified principles and performance indicators to measure process efficiency and identify the most important development areas in product development.

The result of this study is a methodology to identify the most appropriate areas for improvement, verified through a case study. The selection of areas will be based on both qualitative interviews and literature, mainly from Lean product and process development.

\subsection{Creation of a method for measuring systems engineering efficiency}

The purpose of the methodology defined will be to identify and compare process efficiency. Since a standardised baseline for how to measure customer satisfaction was used, the basic structure for structural equation modelling (SEM) was given (Hair et al., 2006). However, we needed to identify the important principles to be measured and the potential performance indicators connected to those principles. In order to accomplish this, a series of business and academic workshops were held. A thorough literature review was also conducted to gain intellectual stimulation. Further, an in-house quality assessment tool used by the case company was used as inspiration. Moreover, regular meetings took place with the internal case company consultants of the team called 'company production system' focusing on the product development process.

The result of this inquiry and the principles identified are presented in the Results Chapter together with its purpose and justification. To accurately map the 18 principles, three to six questions were derived to accurately describe each principle. This process is also clarified in the results section.

\subsection{Case study}

To verify the exploratory approach, a case study was used (Yin, 2008). To gain an in-depth understanding of how business organisations may actually work with process improvement, theoretical sampling (Miles and Huberman, 1994) was applied. As a consequence, a case study was selected employing a strict use of parameters looking at process improvement as a major concern with which to define the cases (Voss et al., 2002). Parameters to look at when selecting the cases were the following:

1 Product and system complexity: the product is of such complexity that no small group of engineers can by themselves have a total overview of the product (e.g., different engineering departments, a large number of individuals and suppliers involved). 
2 Process complexity: not only does the product need to be complex, but the process where it is developed is also full of complexity e.g. a large supplier network, different departments and a preferably stepwise (or stage gate) process, with a multitude of deliverables and integration points.

3 Competitive market: the product and company should act in an environment where large steps towards process efficiency are required in order to remain competitive. In this context, the aerospace industry is an excellent candidate. Within our research network, connected to the Wingquist Laboratory at Chalmers University, one such company existed and was hence selected as the object of our case study.

The case company has a large focus on research and technology development within its organisation which shows that it spends much time and effort on R\&D. Further, the case company is independent which makes it an isolated case, delimited concerning its products and geographic location in relation to competitors, customers and partners.

\subsubsection{Multivariate technique}

The multivariate technique with which to perform the statistical analysis is partial least square (PLS). The PLS analysis is used as a tool by the researchers to gain further knowledge of the datasets collected. To perform the actual calculations, an application called Smart-PLS (Ringle et al., 2005) was used.

The PLS method is a well-established method for management and marketing research (Chin, 1998). PLS is a powerful method of analysis because of the low demands on measurement scales, sample size, and residual distributions. PLS can be used for theory confirmation, as well as for exploratory research. The scope of PLS would be to identify dependencies between variables in order to measure impact on such variables as user satisfaction (Chin, 1995).

\subsubsection{The quantitative case study}

The quantitative study was designed as a PLS-path modelling survey with the hypothesis that satisfaction with the development process may contribute to a variety of performance indicators. The research process was based on a standard quantitative survey method presented in Hair et al. (2006).

Based on customer satisfaction surveys, a questionnaire was designed around 18 principles for efficient product realisation combined with an additional five performance indicators including efficiency, effectiveness, lead-time, cost and quality. A total 74 questions was posed giving about three questions connected to each principle and performance indicator.

The questionnaire was sent out to 324 valid email addresses and resulted in 242 responses. This mailing represented the entire population of engineers and related staff at the company, which makes it a complete sample size. The data collected were first tested using standard missing value analysis (MVA). The final results were calculated as index scores and then path-modelled in SmartPLS. Finally, the index scores were combined with the impacts in the path model to yield a total improvement potential' for each performance. 
In the instructions to the questionnaire, it was stated that all respondents should answer the questions from their own perspective and current position, i.e., how the statement relates to them. It was not specifically asked how engineers work right now nor if a factor or principle was important. A typical question connected to the quality culture principle posed was:

- We identify the cause of potential failure early in the product development process.

The respondents marked on a 1-10 Likert scale if they agreed or disagreed with this statement.

Figure 1 Survey case study (see online version for colours)

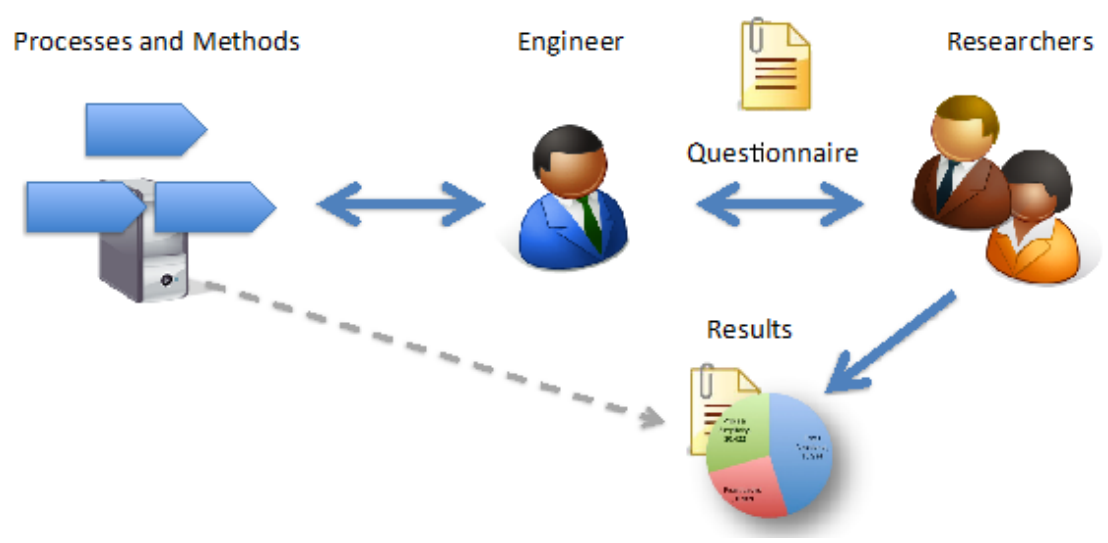

Notes: Engineers are surveyed on their views of the processes. The collective view is then extracted and presented to show improvement potential.

\subsection{Missing value analysis and data quality}

The dataset was tested for a variety of biases and errors. The standard MVA algorithm was used in the statistical IBM SPSS tool. The result showed reasonable standard deviation and missing values.

The data quality was calculated in SmartPLS. The calculated Cronbach alpha and $\mathrm{R}^{2}$ are shown in Table 1. The table shows the significant principles and performance indicators left after the correlations had been calculated. Areas that could not be measured significantly and were removed cannot be given any scores. As a summary of the different scores, Cornbach's alpha is a measurement to indicate the reliability of the areas measured. An alpha number below 0.5 is in general considered unacceptable, whereas numbers above 0.8 are considered good. $\mathrm{R}^{2}$ is the coefficient of determination used to determine how well a regression line fits the data. A value of $\mathrm{R}^{2}$ close to 1 shows a really good fit, and a value close to 0 shows a poor fit (only performance indicators marked by a $\mathrm{P}$ in brackets can be calculated using $\mathrm{R}^{2}$ ). 
Table 1 Survey quality scores (significant factors and performance indicators)

\begin{tabular}{lcc}
\hline Measured areas & $R^{2}$ & Cronbachs $\alpha$ \\
\hline Cross functional work & - & 0.6735 \\
Effective (P) & 0.6762 & 0.5603 \\
Efficiency (P) & 0.6407 & 0.8442 \\
Front loaded PD & - & 0.7371 \\
Innovation/creativity (P) & 0.4053 & 0.8981 \\
Lead-time (P) & 0.3843 & 1 \\
Organisational design & - & 0.8082 \\
Portfolio management & - & 0.5319 \\
Process simplicity & - & 0.7345 \\
Product assurance practices & - & 0.8053 \\
Product cost (P) & 0.2584 & 0.8468 \\
Pull systems & - & 0.6404 \\
Quality (P) & 0.4661 & 0.7271 \\
Satisfaction (P) & 0.6135 & 0.9167 \\
Standardisation and modularisation & - & 0.7513 \\
\hline
\end{tabular}

Notes: The connection between the dependent variables (marked by $\mathrm{P}$ for performance) and independent variables are shown in Figure 3. Only dependent variables have an $\mathrm{R}^{2}$ value.

\subsection{Hypothesis and research questions}

The research approach in this paper is focused on using PLS analysis to analyse and explain patterns in a quantitative data material. The main objective is to identify potential improvement principles connected to positive outcomes in product development to enable management to prioritise improvement projects and identifying potential opportunities for improvement.

The following research questions are to be answered in this paper:

RQ1 What principles and performance indicators are important in the area of process efficiency?

The baseline framework calls for principles and effects that are connected to a set of questions. A standardised generic set of principles are called for that can be used to compare sites and companies. The process targeted is the product development process including interfaces with production except for production itself.

RQ2 Can principles regarding process efficiency be accurately correlated and valued considering their impact on process efficiency?

This question will verify that the principles and analysis methodology provide correct information. In order to give an answer, a case study is required where the tool is tested in reality. We want to identify if the correlation between principles and performance indicators can be used to identify improvement areas. 


\section{Results}

\subsection{Principles of efficient product development}

In order to categorise and measure improvements in the product development process, 18 principles were grouped into four categories and defined. These principles are the result of internal workshops at the case company and are well supported by references to related research. Consequently, it was decided to keep the existing case company name and definitions of principles rather than those used in literature. The principles are loosely based on Liker's (2004) 14 principles as well as Fujimoto's (1999) research.

\subsection{Teamwork modules}

Teamwork includes creating a sound organisation in which all employees are involved in the cross-functional improvement process contributing to achieving company goals and strategic objectives to fully utilise everyone's experience, knowledge and creativity. A foundation for good organisational teamwork is the availability of project management skills, a broad perspective, teamwork skills, and our expanded social network, in addition to boundary spanning skills (Edmondson and Nembhard, 2009).

Within the teamwork module, the following four modules have been identified:

\subsubsection{Goal-oriented team}

Goals for the team need to be defined, visualised and implemented, and activities to sharpen and fulfil them must be managed. Goals are efficiently met by having a clearly expressed management direction with proper empowerment of each team (Bstieler and Hemmert, 2010). To support this module, it is important to work proactively with goals that are aligned throughout the organisation.

\subsubsection{Cross-functional work}

Cross-functional work is established in order to ensure utilisation of the full potential of the organisation. It is important that the organisation establish priorities and proper empowerment among line functions and projects in order for development work to run smoothly. In marketing literature, the relationship between cross-functional teams and innovative performance has been investigated and proven to impact various performance indicators (Luca and Atuahene-Gima, 2006).

\subsubsection{Organisational design}

In order to secure a good teamwork structure, the organisation is designed to fit the needs of the value stream. Important issues for this module include the availability of good career paths for the technical expert and the ability of the organisation to handle deviations and unforeseen circumstances quickly. Adamsson (2007) specifically stresses the need for organisations to be flexible too meet the requirements of a changing workforce. 


\subsubsection{Visualisation and communication}

Visual management provides a clear and common understanding of goals and measures to facilitate decision making and support quick and appropriate improvement measures. Information must be visually available, be posted on walls and boards and the staff must find the information accurate and up-to-date in order for the visual planning system to contribute to improved coordination and communication (Lindlof and Soderberg, 2011).

\subsection{Built-in-quality module}

Building high-quality products require a quality-conscious culture and working with continuous improvements across an extended value chain. A quality-conscious culture needs to permeate the entire organisation. It is essential to involve production as well as other stakeholders early in the development process. There has traditionally been excessive focus placed on teaching tools and techniques whereas the value of tools and techniques derives from an abstract 'quality culture' (Dahlgaard and Dahlgaard-Park, 2006). The following modules have been addressed by the built-in-quality principle:

\subsubsection{Quality culture}

A quality culture basically entails incorporating quality into the shared value and behaviour of individuals. Questions in the questionnaire should specifically target regular contact with the aftermarket discipline, an understanding of customer and product usage, as well as the manufacturing process, including regular visits to production sites.

\subsubsection{Product assurance practices}

Product assurance practices represent an integrated approach by which products that are insensitive to the source of variation may be developed. The questionnaire contains questions on product functionality and the availability of product information. The organisation must also feature methods by which the causes of product failures can be identified.

\subsubsection{Assured start of production}

Assured start of production is an approach to improve performance at the initiation of production, thereby capitalising on the market window of opportunity. Examples of important issues include the clear definition of production start-up and an organisational system for learning from previous product launches.

\subsubsection{Extended value chain}

An extended value chain creates shared knowledge and competence across an entire value chain, thereby decreasing sub-optimisation. Methods should exist to estimate the cost of poor quality. It is also important to involve key stakeholders and suppliers early in the design process. Both long- and short-term aspects of supplier involvement are vital for quality and efficiency of production (van Echtelt et al., 2008). 


\subsection{Just-in-time principles}

The main purpose of the just-in-time philosophy at the case company is to reduce lead-time and create positive effects on many other management aspects, including buffers for stock depletion and over-production avoidance. The principles derived from the Toyota manufacturing system tell us that "only the necessary products at the necessary time in the necessary quantity are manufactured and in addition, the stock on hand is held down to a minimum" (Sugimori et al., 1977). For product development, the identification of bottlenecks to facilitate flow is essential. The following four modules have been selected to assess just-in-time principles:

\subsubsection{Front-loaded product development}

By utilising front-loaded product development whereby alternatives are thoroughly analysed up front, there is less need for rework at the end and higher probability for delivery on schedule. The questionnaire also includes questions on 'firefighting' and the use of milestones in the process.

\subsubsection{Concurrent engineering}

Product development, manufacturing and aftermarket departments concurrently develop products/processes to reduce time-to-market, improve quality and minimise costs. Different organisational functions should work well in parallel.

\subsubsection{Project assurance practices}

Project assurance practices represent an integrated approach to secure the strategic competencies and practices needed to deliver successful projects. An important requirement is for project managers to be skilled and educated in project management.

\subsubsection{Pull systems}

Through the application of a pull system, the required information will be accessible for engineers to utilise whenever necessary. Waiting time should be held to a minimum and the existing information flow should be limited and relevant to fulfil the needs of engineers. A good combination of push and pull initiatives are recommended to achieve a steady flow in production and development processes (Spearman and Zazanis, 1992).

\subsubsection{Resource flexibility}

A resource flexibility approach supports the management of workload volatility. Questions asked in the questionnaire reflect the availability of resources for unplanned activities, as well as a well-balanced budget for projects. Such a resource may also include the application of knowledge in different settings and leveraging this knowledge throughout the organisation (Combs et al., 2011). 


\subsection{Process stability principles}

Process stability means reducing all kinds of undesirable variation and waste during the development processes to avoid late design loops, making the process more predictable and efficient, and creating additional room for innovation. The following five modules are connected to process stability:

\subsubsection{Portfolio management}

Portfolio Management involves making strategic choices and deals with the critical issue of balancing resources available against the number of projects. Questions asked target the cascading effects of delayed projects, as well as conflicts between the functional and project-oriented organisation.

\subsubsection{Standard way of working}

A standard way of working will harmonise processes and reduce variation. By making processes more predictable, there will be additional room for innovation. Standardised and predictable processes should exist within the organisation. Engineers should perceive that there is enough time to be creative to design innovative products.

\subsubsection{Process simplicity}

Process simplicity maximises the efficiency of the value chain by simplifying the information transfer process. Questions asked focus on the establishment of a "continuous improvement" mentality in which non-value-adding tasks are continuously discouraged.

\subsubsection{Product standardisation and modularisation}

Product standardisation and modularisation increase the efficiency in the product development process by reducing complexity. There should exist methods for limiting new part introduction, in addition to common platforms and shared technologies throughout the organisation. Designing a system to adapt and build an architecture supportive of the reuse of components and systems leads to positive effects both in terms of variability and production economics (Engel and Browning, 2008).

\subsubsection{Competence management}

Competence management facilitates the definition of current and future competence needs and the planning to secure these needs in order to create a stable development and manufacturing process. Methods to facilitate knowledge sharing should be in place and accessing the required competence to complete necessary tasks should be easy.

\section{Results from case study}

Our quantitative analysis was performed in three steps and yielded three different results. First, the scores of each principle were calculated and assigned a numeric value. Second, 
impacts were calculated using PLS software. Finally, the overall improvement potential was calculated by combining each score with its respective impact in order to identify the principles with the highest improvement potential, using a simple algorithm presented in this chapter.

\subsection{Scores of principles}

The score of the principle was calculated in SmartPLS (Ringle et al., 2005) by using the PLS-algorithm, with the path weighting scheme setting and mean replacement. In the diagram below, the scores are presented in the same format as the questions were posed, according to a 1-10 Likert scale. The lower principles scored around four and the highest seven. The mean overall score was below the nominal average of 5.5.

Figure 2 Scores of modules (see online version for colours)

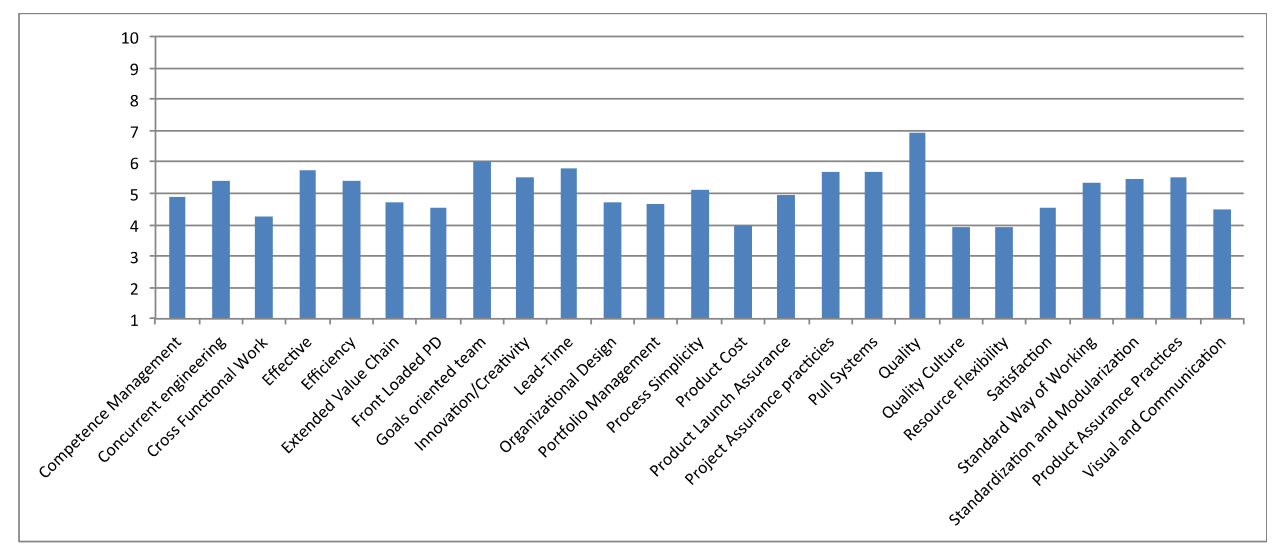

Source: Scores being assessed on a 1-10 Likert scale.

Product quality scored very high at the case company. This result can be traced to the belief that according to customer expectations, the product development process ensures a high quality product. This outcome was confirmed by participants in the workshops.

On the lower end of the score, we find the product cost performance indicator. Signalling that the case company may have difficulties in achieving its targeted product cost. Representatives at the workshop pointed out, that one product in particular was extraordinarily difficult to produce which also led to higher product costs. When isolating this product from other projects in the quantitative dataset, the engineers further confirmed this fact. Engineers working with the difficult product scored lower on the product cost indicator than equivalent engineers working with other products.

Overall, the score diagram of principles indicated that there seemed to be problems with project prioritisation and that there were several late design changes in product development. The story was similar to that involving the product cost principle. In particular, the problematic product scored low on the process stability module. Other issues addressed the quality culture where the contact with aftermarket and production integration scored especially low. Since there seemed to be a lack of resources available for unplanned activities, the resource flexibility principle could be significantly improved. 
The cross-functional work and front-loading modules also scored relatively low, and it was identified in the dataset that frontloading was haunted by postponed milestones and projects that seldom ran on time.

\subsection{Impacts}

In order to look at the network of modules and perceived effects, an impact model was created. In this impact model, independent modules were connected towards dependent performance indicators and their correlation was calculated. The impact model showed impacts among modules with the highest relation to the significant effects. Quality was placed in the middle together with satisfaction to stress the hypothesis that high quality, as well as Satisfaction, leads to the positive effects stated on the right.

Figure 3 Impact model (see online version for colours)

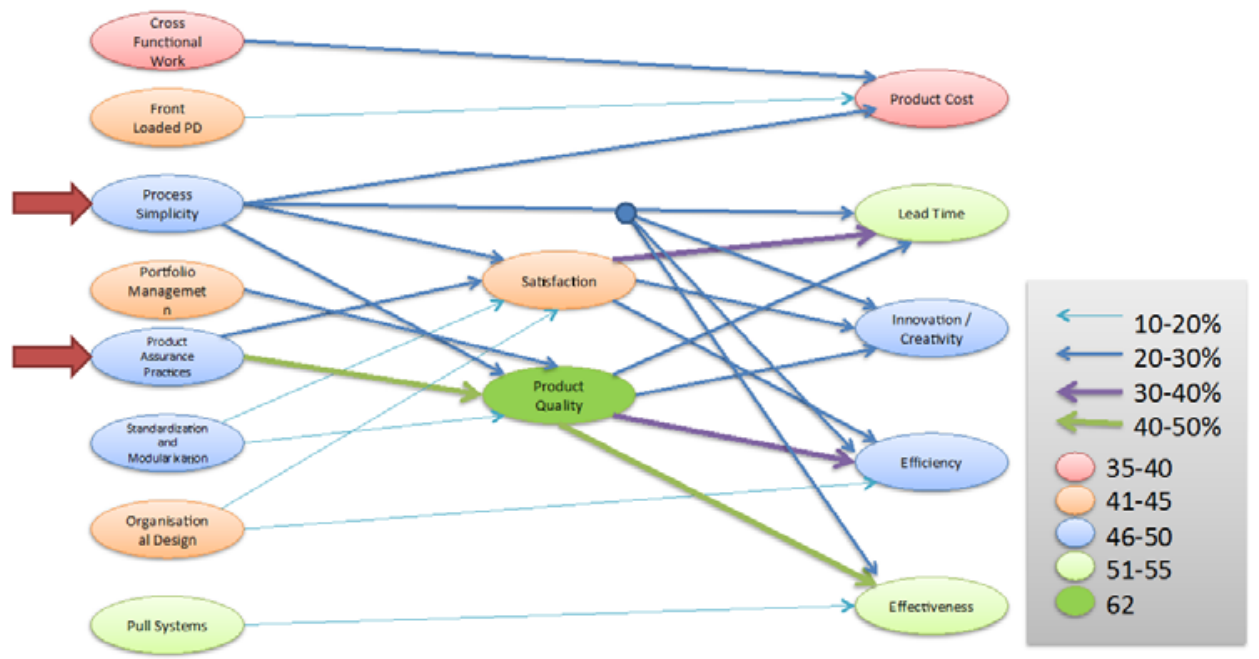

Notes: Connections are modelled in percentages (thicker lines indicate a higher impact). Index values, depicted as circles, are rated from 1-100 and coloured according to legend. A value below 40 is considered low (red) and a value above 51 is considered high (green).

A typically strong correlation between product assurance practices and effectiveness was identified. In other words, engineers clearly see the connection between tools to ensure quality, the actual product quality achieved and the efficiency of the product development process. This outcome was desirable and showed an understanding of attaining a high quality product and the positive effects that come from such results. Since this outcome was combined with a high product quality score, the company showed a quality culture within its development function.

Process Simplicity has strong impact on all important factors, indicating that engineers see a positive connection to simple and applicable process support tools. By interpreting the questionnaire and dataset, our research team concluded that additional work with Kaizen combined with continuous improvements to make knowledge available throughout the organisation were going to have large positive effects on measured performance, with a statistical improvement potential of about $20 \%$. Such a process 
would yield the best impact on future product development and process satisfaction. Strong improvements in this area would lead to higher efficiency and lead-time reduction.

Another major issue would be working proactively identifying potential causes of failure early in product development. One way of doing this, backed up by the workshops, would be to place greater emphasis on visual planning and representation of the process workflow.

In order to reduce product cost, it was advisable to work with the cross-functional principle, in particular the prioritisation of the project portfolio and reducing the number of redesign efforts, possibly by adding resources for unplanned activities. By reviewing the quantitative data, it would also be applicable to the improvement of frontloading aspects.

\subsection{Improvement potential}

In order to simplify the presentation of the results in the previous section, a simple model to calculate an 'improvement potential index' was designed. It indicates that a factor that is rated low in combination with a high impact shows a great improvement potential. The formula for this calculation is:

$$
\text { Improvement Potential }=\text { Impact } \times \frac{10-\text { Index }}{10}
$$

Since impacts are measured in the $0-1$ range and index values in the 1-10 range, a compensation factor should be used.

\subsubsection{Total improvement potential}

The total improvement potential is plotted as a pie chart in which all impacting principles are summarised, making the diagram easier to read compared to the impact model above. When the total improvement potential from all positive effects has been summarised, the diagram in Figure 4 is generated, which clearly shows that the product assurance practices and process simplicity factors have high impacts, as was implicitly shown in the impact diagram.

$$
\text { Total Improvement Potential }=\sum_{\text {All }} \text { Impact } \times \frac{10-\text { Index }}{10}
$$

\subsubsection{Improvement potential on specific effects}

Depending on the effects on which management wants to focus, different principles should be targeted. Figure 4 demonstrated that the Process assurance practices and process simplicity factors had a major total impact, which was also the case with the specific effects (Figure 5). However, if the product cost indicator should be targeted, cross-functional work would be a more important factor to target. 
Figure 4 Total impact on all effects (summarised) (see online version for colours)

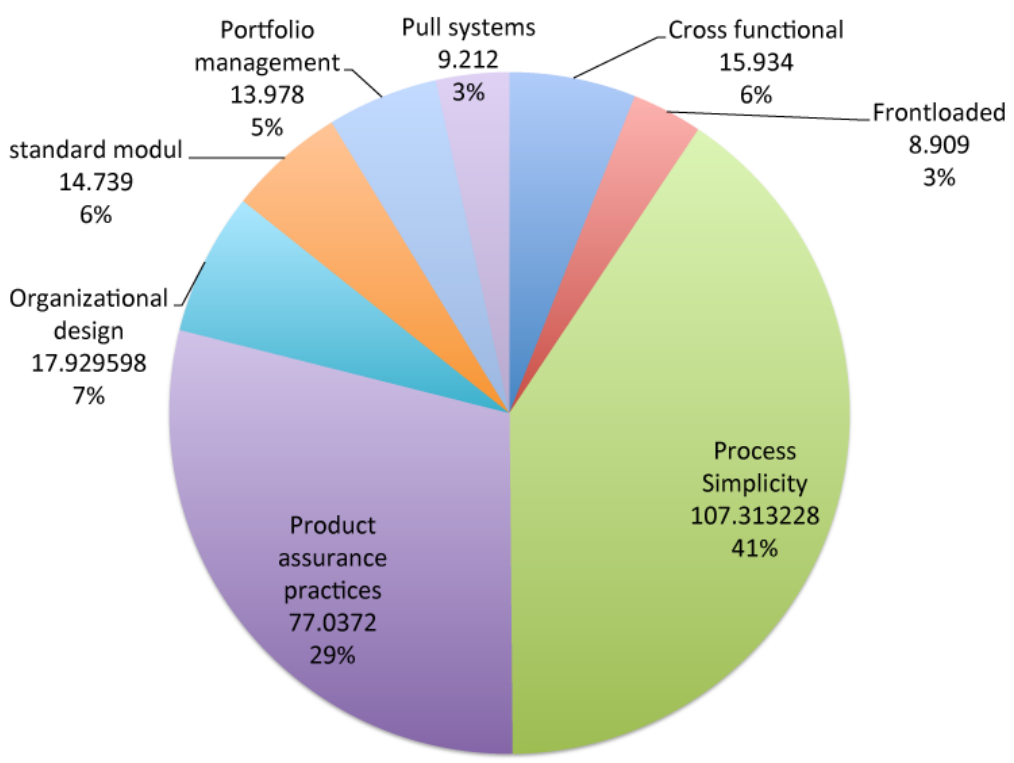

Figure 5 Improvement potential of each principles, (a) impact on leadtime (b) impact on innovation (c) impact on efficiency (d) impact on effectiveness (e) impact on product cost (see online version for colours)

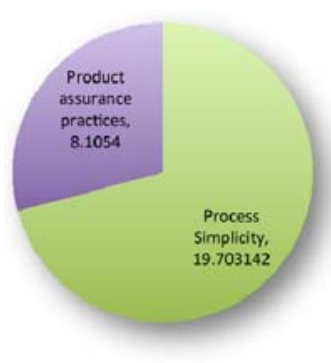

(a)

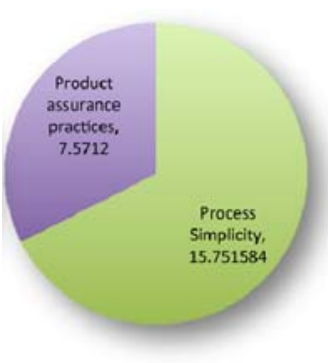

(b)

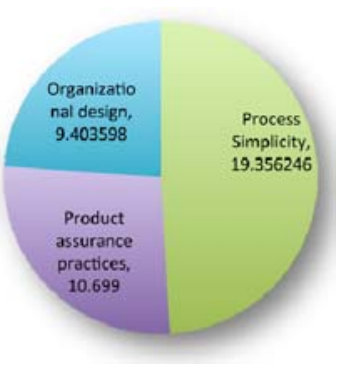

(c)

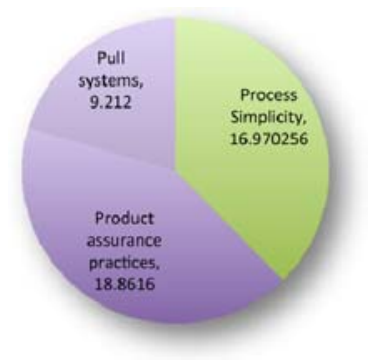

(d)

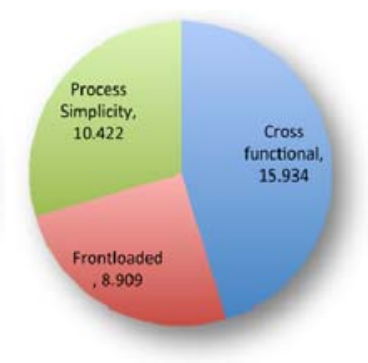

(e)

Note: Indirect impacts are calculated using the product quality and satisfaction effects. 


\section{Discussion of PLS survey tool}

\subsection{Identification of improvement principles}

By utilising a survey-based methodology within a product development company, it is possible to explore and identify potential barriers and opportunities, as shown in this paper. First of all, the survey gives management statistical evidence of the need for improving work procedures and the product development process. This statistical evidence can be useful for management at several levels within a company. For upper management, it gives a good indication of the perceptions that design engineers working in the product development processes consider to be the most important. Management can then direct training and improvement towards these issues. Statistical evidence, graphs and charts can be communicated easily in order to highlight the importance of a specific problem, for example ensuring funding for implementing improvements. In this case, the use of a statistical survey offers the opportunity to prioritise company resources to where end users perceive the greatest needs and benefits to be, or towards the issues that yield the largest effect on efficiency or user satisfaction. The statistical methodology presented provides statistical index values indicating where the problems might be and also shows impacts connected to the goals of the organisation. The approach gains further significance by correlating potential problems to significant gains, such as an increase in quality, efficiency and innovation.

The method presented in this paper highlights problems without offering any solutions to specific problems. This approach can be used to eliminate areas not requiring immediate attention where participating engineers experience a high satisfaction level. This method quickly and significantly narrows down the scope of an improvement project in a cost-effective way. Further studies into the problem areas related to the specific principles are necessary in order to know what needs to be done to improve the situation even though the questionnaire gives an indication of where to start. This search for solutions might involve interviews with people in different functions, for example individuals identified in the questionnaire as particularly dissatisfied or by possibly launching a second and detailed questionnaire that pinpoints the specific issues that were brought up in the initial survey. It could also mean that reorganisation or new methods would need to be incorporated into the process in general and the specific problem would not need to be identified in those cases. Finally, the engineers participating in the survey had the opportunity to comment on each question. These comments generated significant feedback, and further knowledge about the issues at hand can be gained by qualitatively analysing these comments.

\subsection{Surveys as a management tool}

The survey approach presented may possibly be generalised into a method or tool that could be applied to other businesses that operate in a complex process and product environment. The questionnaire is considered to be general and focuses on general management and process issues. The 18 principles for efficient product realisation are applicable to most businesses producing and manufacturing products. The ability to assess the current situation and beliefs among those individuals working with the 
processes could give management a powerful tool to direct their improvement efforts to where they are most urgently needed and valued by their employees.

\subsection{Better understanding of the user perspective}

The survey used in this study enables each engineer working in product development to make his/her voice heard. Such feedback could yield significant benefits about how an introduction or improvement project is progressing. In Malvius et al. (2007a, 2007b), it was stated that from a management perspective, it is easy to make a mistake by listening to a few loud opinions rather than gathering a rich and balanced assessment of the general user perspective. It is also difficult to identify engineers who are representative of the workforce in order to perform interviews capturing the user perspective. This approach gives a richer data bank of responses based on the whole population compared to such instruments as self-assessment and qualitative interview studies.

As a criticism, it might be easier to directly provide design engineers with a list of potential improvements that management and experts have identified and ask users to rank them. Such an approach would give end users a very direct way of prioritising their problems more quickly than the questionnaire-based survey presented in this paper. As an answer to such criticism, this survey approach identifies problems rather than solutions and the engineers participating in the survey do not need to be aware of the solutions in order to generate index values and impacts. Such an alternative scenario might identify problem areas that are difficult to pinpoint by posing direct questions.

\subsection{Assessing the product realisation process - benchmarking}

A helpful way by which the results may be utilised is to perform a benchmark study using the data from the survey. Such benchmarking could conceivably be performed between sites within an organisation or between companies. When comparing different departments, principles that are more or less beneficial might be identified and the various departments could thus try to find out reasons for the difference and learn from each other. For example, it may be discovered that organisational learning is much better in one organisation and that this organisation also experiences higher user satisfaction; these results could then be used to motivate engineers in another department to implement similar procedures and tools. It may be found that the tasks performed in the various departments differ in such a way that they cannot learn or adapt the processes used from each other; but even in such instances, dependencies could be identified. For example, one department may mainly push information and may not experience any difficulties with cross-functional collaboration but since the information could be difficult to use or be poorly adapted to its process or IT tools, the department receiving the information may experience problems. In this scenario, the dissatisfaction in one department might be used to improve the workflow in another department in order to improve overall satisfaction. The same approach could be used to compare the business organisation with others within the same industry or with companies from other business areas. For example, the banking industry is very different from the aerospace business but may have processes that are quite efficient and that may be modified to serve the aerospace business. A comparison between such industries would open up possibilities of finding truly innovative solutions to process related problems. 


\subsection{Continuous improvement and refinement}

New tools and methods are continuously being developed and introduced into product development, including the introduction of new support and IT tools to aid administrative tasks. Some of these changes are regarded as positive by users but many also resist change. The connection to lean principles and positive effects makes it possible to motivate and assess changes in an organisation more efficiently.

When performing surveys regularly, it may also be possible to make long-term goals and work proactively with selected principles. A goal might be formulated that a new support tool for cross-functional collaboration could lead to $10 \%$ more satisfied users; this type of goal may mean that a survey would be launched prior to the introduction followed by another a few months later. For longer-term goals, it may be reasonable to set targets that perceived process efficiency should increase. Under those circumstances, management would preferably target areas where the principles have the highest improvement potential.

\subsection{Risk and bias inherent in survey methodology}

As with all research approaches, it is difficult to ensure unbiased results. The survey design had an initial built-in bias because it had been established within an existing set of company principles and questions. However, to counter the initial pragmatic approach, an external researcher was involved in the process and changes were made both in how the questions were designed and what was asked in comparison to the baseline methodology of the company. Starting with the research community and then looking at specific business interests, not the other way around, could have presented a more reliable or unbiased approach. Note that the intention of the questionnaire was to measure perception and opinion. The questionnaire and methodology should always be used in combination with knowledgeable experts to ensure that a problem was an actual rather than a perceived problem before taking large-scale action to solve an issue. Education or information to employees might be a potential solution by which a "perceived" problem area could be fixed.

Considering the design of the questionnaire, the questions were designed to be answered relatively quickly and a lot of effort was spent to consistently pose questions positively, with 10 as the best answer. An imminent drawback to reduce the potential for misunderstanding was to make the questions less specific. The survey tool hence became an indicator that something was wrong rather than pointing out exactly what went wrong. The latter type of answers would more reliably be given by qualitative interviews focusing on one or two 'problem areas'.

To eliminate structural errors, several statistical tools may be used. The Cronbach alpha and $\mathrm{R}^{2}$ indicate reliability. However, a drawback with the selected methodology (SEM PLS) is that it is difficult to measure model-fit or potential problems using the model. A rule of thumb that exists described by (Chin, 1995) essentially states that you need ten times the number of questionnaire answers than indicators. Consequently, a study with 15 indicators would require more than 150 reliable answers, which is fulfilled with almost the double number of questionnaire answers, 247. 


\subsection{Potential improvements}

As with all research studies, it is important to inquire into the benefits and drawbacks of a particular method. Could it be that researchers delve so far into the figures and principles that the engineers perceiving the problems are left out? It must be made clear that performing a survey is a complement to other tools and studies used to improve the internal performance of a company. In the case study, a qualitative interview study was performed in parallel in order to verify business requirements.

The method could also be challenged on the basis that experts and managers know the optimal solution. Even in this instance, it is best to employ the survey as a complementary tool; many phenomena can be identified in a questionnaire, but they are all going to be explicit needs. Needs that are taken for granted and those which they did not know they had will not be explicit. Sometimes management must know the optimal solutions and the decisions that comply with company strategies. However, even in those instances, it is better for management to know what their employees think rather than relying on second- hand information from various internal sources.

Further studies are called for about actions to be taken in order to settle principles surrounded by uncertainty. As mentioned before, the survey does not pinpoint any specific problems but rather problem areas inherent in the principles. In the case study, the information regarding the difficulties is transferred to management and the entire organisation through several presentations. It is, however, up to the organisation to decide which actions to take in order to improve its development processes. When data have been collected over time and more has been learned about the specific organisation measured, it will probably be possible to act with greater confidence to arrive at direct action plans for dealing with the problems identified.

The way in which the survey is presented and launched in an organisation can also be improved. The study should be anchored at the management level closest to the practicing engineer. In the case study, only the support of top management was assured, which meant that some group managers were not informed. Further information could be obtained by attending group meetings of engineers and ensuring that time was allocated for each engineer to fill out the questionnaire.

\section{Conclusions}

This paper has presented a methodology for a quantitative approach with which to identify the improvement potential inherent in a development process verified by a case study. In the case study, 247 engineers participated in assessing a set of 18 principles important to product development. The use of a statistical method gave an understanding of the bottom-up perspective in an organisation. The study was validated through a workshop with top management, including self-assessments, and was concluded by four separate presentations involving the company engineers.

The case study showed that the new methodology could be performed quickly with great benefits for the case company compared to self-rating methods. Our quantitative dataset is rich and can be used for the following tasks:

- identifying best practices

- comparing various company departments or sites 
- $\quad$ identifying the highest needs from a user perspective

- $\quad$ presenting a baseline for continuous improvements

- balancing user and management perspectives.

Further, the questionnaire gave employees the motivation to contribute to process improvement and suggestions. Comments from respondents gave valuable insights into the process and further validated the quantitative approach.

Based on the case study performed, it was possible to objectively assess and identify principles that needed additional attention. From the case study, the following principles demonstrated the greatest improvement potential:

- $\quad$ process simplicity: flow and batch sizes, visual control, pull system

- $\quad$ product assurance practices: project management, project cost, quality

- $\quad$ organisational design: chief engineering concept, different career paths, work rotation, etc.

\section{Acknowledgements}

Carried out at the Wingquist Laboratory VINN Excellence Centre within the Area of Advance Production at the Chalmers University of Technology, Gothenburg, Sweden, this study was supported by the Swedish Governmental Agency for Innovation Systems (VINNOVA). The VINNOVA support is gratefully acknowledged.

\section{References}

Adamsson, N. (2007) Interdisciplinary Integration in Complex Product Development, PhD, KTH-Royal Institute of Technology, Stockholm.

Bstieler, L. and Hemmert, M. (2010) 'Increasing learning and time efficiency in interorganizational new product development teams', Journal of Product Innovation Management, Vol. 27, No. 4, pp.485-499.

Chin, W.W. (1995) 'Partial least squares is to LISREL as principal components analysis is to common factor analysis', Technology Studies, Vol. 2, No. 2, pp.315-319.

Chin, W.W. (1998) 'The partial least squares approach for structural equation modeling', Modern Methods for Business Research, pp.295-336, Lawrence Erlbaum Associates Publishers, Mahwah, NJ, USA.

Chiva, R. and Alegre, J. (2009) 'Investment in design and firm performance: the mediating role of design management', Journal of Product Innovation Management, Vol. 26, No. 4, pp.424-440.

Combs, J.G. et al. (2011) 'The role of resource flexibility in leveraging strategic resources', Journal of Management Studies, Vol. 48, No. 5, pp.1098-1125.

Dahlgaard, J.J. and Dahlgaard-Park, S.M. (2006) 'Lean production, Six Sigma quality, TQM and company culture', The TQM Magazine, Vol. 18, No. 3, pp.263-281.

Edmondson, A.C. and Nembhard, I.M. (2009) 'Product development and learning in project teams: the challenges are the benefits', Journal of Product Innovation Management, Vol. 26, No. 2, pp.123-138. 
Engel, A. and Browning, T.R. (2008) 'Designing systems for adaptability by means of architecture options', Systems Engineering, Vol. 11, No. 2, pp.125-146.

Fujimoto, T. (1999) The Evolution of a Manufacturing System at Toyota, Oxford University Press, UK.

Hair, J. et al. (2006) Multivariate Analysis, 6th ed., Prentice Hall, Upper Saddle River, New Jersey, USA.

Liker, J.K. (2004) The Toyota Way 14 Management Principles from the World s Greatest Manufacturer, McGraw-Hill, USA.

Lindlof, L. and Soderberg, B. (2011) 'Pros and cons of lean visual planning: experiences from four product development organisations', International Journal of Technology Intelligence and Planning, Vol. 7, No. 3, pp.269-279.

Luca, L.M.D. and Atuahene-Gima, K. (2006) 'Market knowledge dimensions and cross-functional collaboration: examining the different routes to product innovation performance', Journal of Marketing, Vol. 71, No. 1, pp.95-112.

Malvius, D. et al. (2007a) 'Balancing operational and strategic impacts on information management', IDETC/CIE 2007, Proceedings of the ASME 2007, Las Vegas, USA, DETC2007-35438.

Malvius, D. et al. (2007b) 'Shifting lead as PLM introduction strategy', ICPLM'07, Bergamo, Italy, pp.179-188.

Miles, H. and Huberman, M. (1994) Qualitative Data Analysis: A Sourcebook, Sage Publications, Beverly Hills, CA.

Rhodes, D.H. et al. (2009) 'Systems engineering leading indicators for assessing program and technical effectiveness', Systems Engineering, Vol. 12, No. 1, pp.21-35.

Ringle, C.M. et al. (2010) 'Response-based segmentation using finite mixture partial least squares theoretical foundations and an application to American customer satisfaction index data', in Stahlbock, R. et al. (Eds.): Data Mining, Annals of Information Systems, Vol. 8, pp.19-49, Springer US, USA.

Ringle, C.M., Wende, S. and Will, A. (2005) SmartPLS 2.0.M3, SmartPLS, Hamburg [online] http://www.smartpls.com.

Spearman, M.L. and Zazanis, M.A. (1992) 'Push and pull production systems: issues and comparisons', Operations Research, Vol. 40, No. 3, pp.521-532.

Sugimori, Y. et al. (1977) 'Toyota production system and Kanban system materialization of just-in-time and respect-for-human system', International Journal of Production Research, Vol. 15, No. 6, pp.553-564.

van Echtelt, F.E.A. et al. (2008) 'Managing supplier involvement in new product development: a multiple-case study', Journal of Product Innovation Management, Vol. 25, No. 2, pp.180-201.

Voss, C., Tsikriktsis, N. and Frohlich, M. (2002) 'Case research in operations management', International Journal of Operations and Production Management, Vol. 22, No. 2, pp.195-219.

Yin, R.K. (2008) Case Study Research - Design and Methods, Sage Publications, Thousand Oaks, CA. 